\title{
The Relationship between Oxygen Saturation and Color Alteration of a Compromised Skin Flap: Experimental Study on the Rabbit
}

\author{
Theddeus O. H. Prasetyono ${ }^{1}$, Senja Adianto ${ }^{2}$ \\ ${ }^{I}$ Division of Plastic Surgery, Department of Surgery, Faculty of Medicine, University of Indonesia and Cipto Mangunkusumo Hospital, \\ Jakarta; ${ }^{2}$ Department of Surgery, Dustira Army Hospital, Cimahi, Indonesia
}

Background The aim of this study was to collect important data on the time of oxygen saturation change in relation to skin flap color alteration using non-invasive pulse oximetry to evaluate its ability to provide continuous monitoring of skin flap perfusion.

Methods An experimental study on the monitoring of blood perfusion of 20 tube-island groin flaps of 10 male New Zealand rabbits was performed using pulse oximetry. The animals were randomly assigned to one of two groups representing a blockage of either arterial or venous blood flow. The oxygen saturation change and clinical color alteration were monitored from the beginning of vessel clamping until the saturation became undetectable. The result was analyzed by the t-test using SSPS ver. 10.0.

Results The mean times from the vessel clamping until the saturation became undetectable were $20.19 \pm 2.13$ seconds and $74.91 \pm 10.57$ seconds for the artery and vein clamping groups, respectively. The mean time of the clinical alteration from the beginning of vein clamping was $34.5 \pm 11.72$ minutes, while the alteration in flaps with artery clamping could not be detected until 2.5 hours after clamping.

Conclusions The use of neonate-type reusable flex sensor-pulse oximetry is objective and effective in early detection of arterial and vein blockage. It provides real-time data on vessel occlusion, which in turn will allow for early salvaging. The detection periods of both arterial occlusion and venous congestion are much earlier than the color alteration one may encounter clinically.

Keywords Free tissue flaps / Oxygen consumption / Oximetry

\author{
Correspondence: \\ Theddeus 0 . H. Prasetyono \\ Division of Plastic Surgery, \\ Department of Surgery, \\ Faculty of Medicine, University of \\ Indonesia and Cipto Mangunkusumo \\ Hospital, Medical Staff Building 4th \\ floor Jl. Diponegoro, 71 Jakarta 13410, \\ Indonesia \\ Tel: $+62-817858899$ \\ Fax: +62-21-3905556 \\ E-mail: teddyohprasetyono@yahoo. \\ com
}

\begin{abstract}
This article was presented in part at the 10th Annual Scientific Meeting of the Indonesian Association of Plastic Surgeons Bali, Indonesia, May 22-23, 2006 and at the 10th International Congress of the International Federation of Societies for Surgery of the Hand (IFSSH), on March 2007 in Sydney, Australia.
\end{abstract}

No potential conflict of interest relevant to this article was reported.

\section{INTRODUCTION}

Monitoring of flap circulation in the postoperative period is one of essential methods for determining the success of a free flap procedure. When early changes in flap perfusion with regard to a vascular compromise are detected, salvaging can be done im- mediately to re-explore and revise the vessel anastomosis [1]. Unfortunately, it is a common practice that surgeons rely only on clinical observations to monitor flap viability, which are mainly based on skin color, temperature, turgor, and the capillary refill or back flow observed through the skin. It can be difficult to use such simple conservative clinical monitoring as a basis for decid- 
ing whether to re-explore the vessel anastomosis. Many doctors have experienced struggling to re-explore a vessel anastomosis in an attempts to salvage a dying flap after encountering questionable early changes observed clinically on the skin paddle. Such early changes, especially the change in skin color, usually provoke the salvaging procedure some time after it is needed and often too late for it to be performed [1-5].

A flap monitoring tool, which is effective, reliable, and easy to use, and easy to interpret, would be helpful for salvaging vascularly compromised flaps. Effective free flap monitoring through a noninvasive device would be preferable to the conventional technique mentioned above or the use of invasive Doppler flowmetry and an implantable microcatheter oxygen sensor. The data obtained from the monitoring device has to be objective, reliable, and accurate in providing real time data, and easy to interpret. Noninvasive special reflection sensor pulse oximetry [6] is an innovative option following the use of completely standard operating theatre equipment, as previously reported $[7,8]$.

Despite the deliberate use of pulse oximetry in monitoring free flap perfusion, there is still a lack of important data concerning its accuracy in providing real-time information on arterial and venous flow. This study aimed to evaluate the ability of pulse oximetry to provide continuous monitoring of skin flap perfusion by comparing the time of saturation change with skin flap color alteration using reusable neonate flex sensors and a portable-sized monitor.

\section{METHODS}

An experimental study was conducted on ten New Zealand white rabbits $(2,000-2,500 \mathrm{~g})$, which were housed and treated in an animal laboratory. The animals were anesthetized with an intramuscular injection of ketamine sulfate of 20 to $40 \mathrm{mg} / \mathrm{kg}$ and ether inhalation. After shaving the hair of the abdominal walls, the site was cleansed with $10 \%$ povidone-iodine solution followed by $70 \%$ alcohol and draped sterilely.

A $4 \mathrm{~cm} \times 5 \mathrm{~cm}$ hexagonally shaped fasciocutaneous island groin flap was marked, dissected, and raised. Both sides of the groin were used to obtain 20 flaps. The superficial inferior epigastric artery and vein were identified and separated from one another. A small incision was also added at the groin to clearly expose the artery and vein without undue tension. The flap was then sutured into a tube shape and the proximal portion of the flap was sutured to the original site at two points, while its distal edge was fixed to a metal bar that functioned as a stabilizer and hanging device (Fig. 1A). The sensor probe was placed on the outside of the middle part of the flap, with minimal pressure, and was fixed with 1-inch-wide paper tape (Micropore, 3M, St. Paul, Minnesota, USA), preserving the distal part to be left open (Fig. 1B). The vessel was not yet clamped until stable oxygen saturation ( $\geq 95 \%)$ had been observed for a few minutes to allow the flap to reach its best flow.

Each animal was classified into one of two groups: one to represent a blockage of the arterial blood flow and the other representing a blockage of venous blood flow. In the five animals of group A, the artery was clamped with a microsurgical vessel clamp, and then the saturation change and clinical alteration were observed and recorded. The saturation change referred to the timing when the oxygen saturation became undetectable. The clinical alteration referred to the color change. Both of the observations regarding the timing were performed with the use of a stopwatch. The observation was also documented with a Fig. 1. The groin flap and oxygen saturation monitor

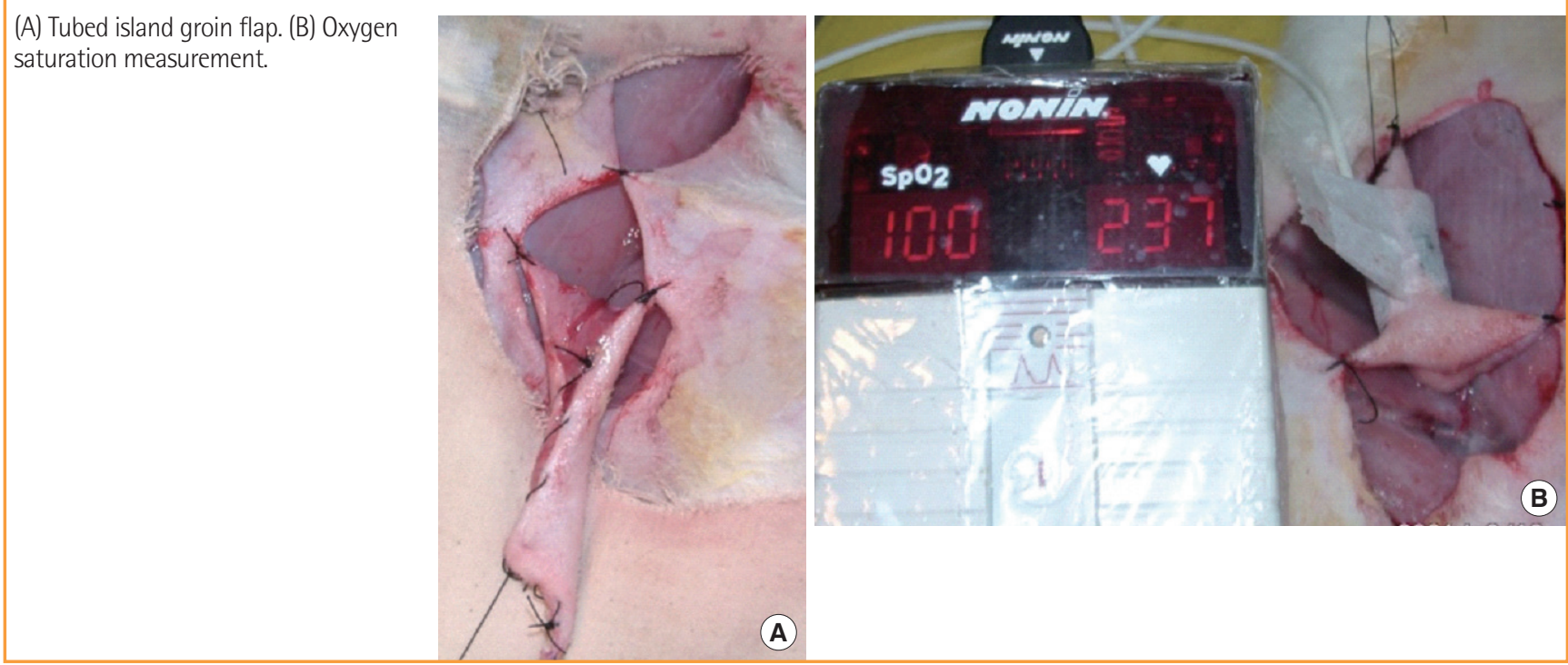


digital camera every 15 minutes for a 2.5 hour period of observation. The photographs were standardized with the same focus, distance, and lighting. In the other five animals of group B, the vein was also clamped with a microsurgical vessel clamp, and then the saturation change and clinical alteration were recorded as in group $\mathrm{A}$.

At the end of the observation time of group A (artery clamping), the vessel clamp was removed and we let the blood flow back into the flap for a few minutes until maximal stable oxygen saturation was achieved ( $\geq 95 \%$ ). Finally, the flap was sutured back to its original position. The flaps of group B would have received the same treatment, but as it had been predicted that thrombus might have blocked the vein flow, they were discarded after 2.5 hours of observation, and the wound in each was primarily closed. The data was analyzed with a t-test using SPSS ver. 10.0 (SPSS Inc., Chicago, IL, USA).

The instruments used were 1) a reusable flex sensor: Neonatal Flex System type 8001J (Nonin Medical Inc., Plymouth, MN, USA), 2) a monitor: Digital Handheld Pulse Oximeter; type 8500 (Nonin), 3) a stopwatch: Diamond DM3-100, 4) a digital camera: Casio QV- 2900 UX, and 5) a semiopen inhalation anesthetic instrument for small animals.

\section{RESULTS}

All of the animals survived without a single dropout. Table 1 shows the records of the observations after artery and vein clamping. The mean times from vessel clamping until the saturation became undetectable were $20.19 \pm 2.13$ and $74.91 \pm 10.57 \mathrm{~s}$ for artery and vein clamping, respectively (Table 1 ). These data were analyzed for normality with the Kolmogorov-Smirnov test, which revealed an abnormal data distribution $(\mathrm{P}=0.022)$.

Table 1. Time of saturation change (the timing of the oxygen saturation when it became undetected)

\begin{tabular}{|lcc|}
\hline \multirow{2}{*}{ No. of sample } & \multicolumn{2}{c|}{$\begin{array}{c}\text { Timing of oxygen saturation when it } \\
\text { became undetected }\end{array}$} \\
\cline { 2 - 3 } & Artery clamping (sec) & Vein clamping (sec) \\
\hline 1 & 18.88 & 89.84 \\
2 & 23.67 & 69.30 \\
3 & 18.06 & 63.28 \\
4 & 18.99 & 61.23 \\
5 & 18.37 & 65.64 \\
6 & 23.93 & 83.12 \\
7 & 19.73 & 76.34 \\
8 & 18.65 & 83.12 \\
9 & 20.56 & 69.17 \\
10 & 21.10 & 88.06 \\
Mean \pm SD & $20.19 \pm 2.13$ & $74.91 \pm 10.57$ \\
\hline
\end{tabular}

Data were transformed with $\sqrt{x}$ methods, and then its normality was analyzed again, revealing a normal distribution $(\mathrm{P}=0.09)$. Finally, with t-test analysis there was a significant difference between the groups $(\mathrm{P}=0.00)$.

It was difficult to determine the timing of the clinical alteration of the flaps in group A. The color of the flaps after artery clamping appeared not to change from the beginning of the clamping to 150 minutes (Fig. 2). On the other hand, the color change observed on the flaps of group B was much clearer after vein clamping (Fig. 3). The mean time of the clinical alteration after the beginning of vein clamping was $34.5 \pm 11.72$ minutes (Table 2 ).

\section{DISCUSSION}

Doppler flowmetry, which usually has the advantage of the ability to use the microprobe encircling the vein distal to the anastomotic site, may provide real-time data based on the sound of the blood flow turbulence. It sounds an alarm when the vein flow stops. Similarly, the use of a microcatheter sensor to measure tissue oxygen tension continuously may be an excellent tool for monitoring flap viability [9]. However, in fact, both of the devices are invasive in nature and are much more expensive than pulse oximetry with a reusable sensor. Therefore, pulse oximetry, which is reliable and easy to interpret, is still a more favorable option.

Certain biological data of rabbits, such as blood pressure (90-130/60-90 mm Hg), erythrocytes $\left(5.0-8.0 \times 10^{6} / \mathrm{mm}^{3}\right)$, hemoglobin ( $8-17 \mathrm{~g} / \mathrm{dL})$, and hematocrit (31\%-50\%), are comparable to those of humans [10]. In addition, the rabbits' arteries and veins were large enough to allow sufficient vessel preservation under loupe magnification. These characteristics

\section{Fig. 2. Color changes on artery clamping}

(A) At 0 minute. (B) At 150 minutes.

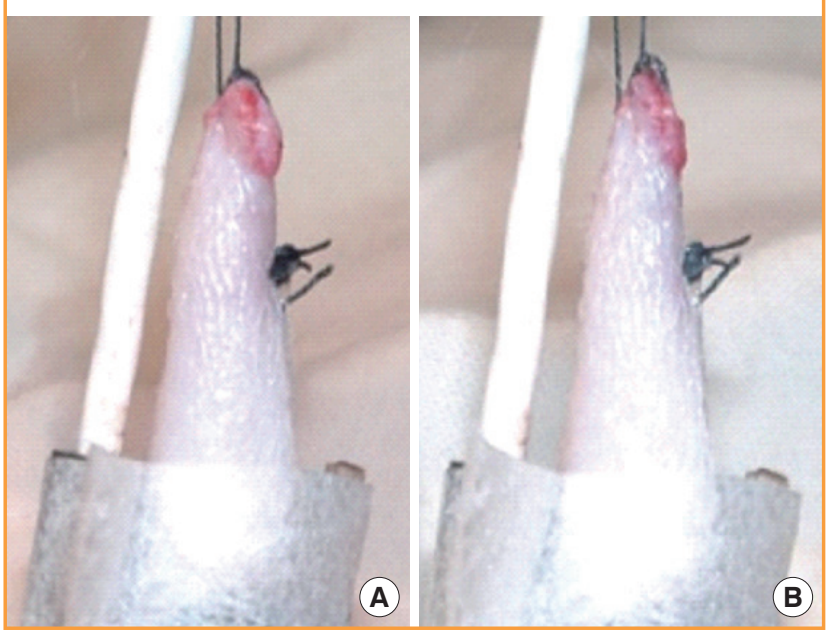




\section{Fig. 3. Color changes on vein clamping}

(A) At 0 minute. (B) At 15 minutes. (C) At 30 minutes. (D) At 150 minutes.

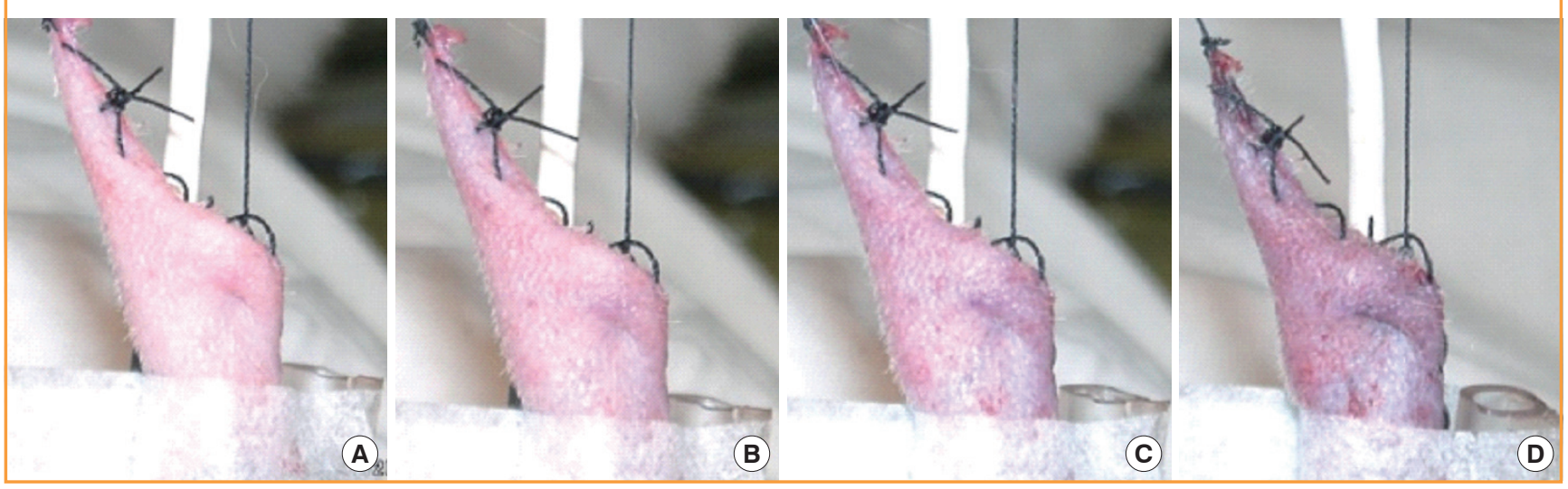

Table 2. Time of clinical alteration (the timing of flap color change)

\begin{tabular}{|lcc|}
\hline \multirow{2}{*}{ No. of sample } & \multicolumn{2}{c|}{ The timing of flap color change } \\
\cline { 2 - 3 } & Artery clamping (min) & Vein clamping (min) \\
\hline 1 & Difficult to be measure & 30 \\
2 & & 45 \\
3 & 15 \\
4 & 45 \\
5 & 30 \\
6 & 45 \\
7 & 45 \\
8 & 30 \\
9 & 15 \\
10 & 45 \\
Mean \pm SD & $34.5 \pm 11.72$ \\
\hline
\end{tabular}

justify performing the study on a rabbit model. The groin flap in a rabbit is large enough in size that it has sufficient space for a probe to be placed. The neonate-type reusable flex sensor pulse oximeter can be readily applied on the tubed island groin flap. A description of the type of flap chosen, its size, and its saturation limit appear in a previous study performed by Khot et al. [11].

In this study, the use of neonate-type reusable flex sensor-pulse oximetry on a tubed groin flap was intended to monitor flap perfusion and detect as early as possible any blockage of blood flow either in the artery or vein. In the event of artery clamping, undetected oxygen saturation appeared in a very short time (an average of $20.19 \pm 2.13$ seconds) according to the monitor. On the other hand, oxygen saturation decreased gradually during vein clamping and became undetectable in an average of $74.91 \pm 10.57$ seconds. Although the timing of the two is significantly different, this is not clinically significant.

Apparently, during the period of vein clamping, oxygen saturation never decreased to less than $85 \%$ until it became suddenly undetectable. This finding is clinically important, as we understand that the flap is still being nourished by the artery. At some point, the arterial flow stops and oxygen saturation falls to an undetectable level. According to Graham et al. [12], in monitoring a replanted finger, saturation of less than $85 \%$ indicates vein occlusion, which means that the occlusion has been happening at a significant level for a long period of time. With regard to toe to finger transfer, this has also been confirmed by Jones and Gupta [13], who stated that oxygen saturation that is far below the oxygen saturation of any control finger for a long time indicates thrombosis in vein anastomosis.

The results of this study provide more precisely objective data regarding the timing of blood vessel compromise. In comparison with the data on clinical color change, an alteration in oxygen saturation gives much more timely alarm information to the clinician. Vein flow blockage, which is commonly a result of thrombosis, will only appear after 34.5 minutes, while the saturation monitor indicates the blood flow stops in only 75 seconds. In a real clinical situation, a color change may take much longer to appear and is frequently difficult to assess. Just after 2.5 hours, extensive venous congestion appeared in the flap, as it can be seen in Fig. 3D. The result of this study confirms the alarming data that significant color alteration does not appear until at least 2.5 hours after arterial flow has stopped. This difficult clinical situation may be easily detected with the use of pulse oximetry, which can detect the arterial blockage just in 20 seconds.

The observation of the clinical color alteration was not designed to be performed blindly, as the clinical pictures were also taken (by the second author) serially every 15 minutes after the vessel clamping. If needed, the photos could be easily used for further study by asking blinded reviewers to evaluate the flap color given in a series of respective samples. Furthermore, the 
authors did not anticipate in advance that there would not be any color change due to arterial clamping, let alone until 2.5 hours of observation. A hundred and fifty minutes might be considered too long for realizing that the arterial flow is being blocked in a real clinical situation. By that time, no more oxygen or nutrition would be being distributed to the flap, while undoubtedly the tissue and cells would be suffering from anaerobic metabolism leading to tissue necrosis. This study showed that in just $20 \mathrm{sec}-$ onds, the pulse oximetry would alarm the doctor in the event of arterial occlusion.

The criteria used for detecting any color change was with reference to the baseline color at the time when the vessel clamp was clipped on. For any possible color changes, either due to artery or vein blockage in a real clinical situation, the authors agreed to refer to the baseline skin flap color, which is recorded photographically right after completing the vessel anastomosis. If the duration of observation were longer, any potential color change would be more prominent as time passed. It would indeed be difficult to determine the "cut-off point" of the color change using any standard such as a chart of colors because of the immense variation in human skin color. This might be a new domain for further study, answering the question of whether a percentage color change could be deemed a "cut-off point" in the skin color change for a given spectrum of color.

This study has a limitation with regard to application to real clinical settings, as it is widely accepted that pulse oximetry requires a thin flap that can be transilluminated. Most skin flaps have a flat surface in which it might be difficult to apply the sensor-pulse oximetry, but the authors used a neonate-type of flex sensor pulse oximetry, which only needed a small area of flap for the analysis.

In summary, the results of this study indicate that oxygen saturation monitoring can be a reliable tool for detecting early artery or vein compromise before flap color changes take place clinically. The use of a neonate-type reusable flex sensor pulse oximeter is objective and effective in early detection of arterial and vein blockage. It provides real-time data on vessel occlusion, which in turn will allow for an early salvaging procedure. The detection periods of both arterial occlusion and venous congestion are much earlier than the color alteration one may encounter clinically.

\section{REFERENCES}

1. Buncke HJ. Monitoring. In: Buncke HJ, editor. Microsurgery transplantation-replantation, an atlas-text. Philadelphia: Lea \& Febiger; 1991. p.715-21.

2. Daniel RK, Kerrigan CL. Principles and physiology of skin flap surgery. In: McCarthy JG, editor. Plastic surgery. Philadelphia: WB Saunders Company; 1990. p.318-23.

3. Shenaq SM, Sharma SK. Principles of microvascular surgery. In: Grabb WC, Smith JW, Aston SJ, editors. Grabb and Smith plastic surgery. 5th ed. Philadelphia: Lippicott-Raven; 1997. p.73-7.

4. Shaw WW, Khouri RK. Clinical microvascular surgery and free tissue transfers. In: Smith JW, Aston SJ, editors. Grabb and Smith's plastic surgery. 4th ed. Boston: Little, Brown and Company; 1991. p.1029-52.

5. McGregor AD, McGregor IA. Fundamental techniques of plastic surgery: and their surgical applications. London: Churchill Livingstone; 2000.

6. Strauss JM, Neukam FW, Krohn S, et al. Postoperative monitoring of microvascular flap repair with pulse oximetry: initial experience. Handchir Mikrochir Plast Chir 1994;26:80-3.

7. Lindsey LA, Watson JD, Quaba AA. Pulse oximetry in postoperative monitoring of free muscle flaps. Br J Plast Surg 1991;44:27-9.

8. Menick FJ. The pulse oximeter in free muscle flap surgery. "A microvascular surgeon's sleep aid". J Reconstr Microsurg 1988;4:331-4.

9. Hirigoyen MB, Blackwell KE, Zhang WX, et al. Continuous tissue oxygen tension measurement as a monitor of free-flap viability. Plast Reconstr Surg 1997;99:763-73.

10. Yelderman M, New W Jr. Evaluation of pulse oximetry. Anesthesiology 1983;59:349-52.

11. Khot MB, Maitz PK, Phillips BR, et al. Thermal diffusion probe analysis of perfusion changes in vascular occlusions of rabbit pedicle flaps. Plast Reconstr Surg 2005; 115:1103-9.

12. Graham B, Paulus DA, Caffee HH. Pulse oximetry for vascular monitoring in upper extremity replantation surgery. J Hand Surg Am 1986;11:687-92.

13. Jones NF, Gupta R. Postoperative monitoring of pediatric toe-to-hand transfers with differential pulse oximetry.J Hand Surg Am 2001;26:525-9. 